\title{
EFFICACY OF TERIPARATIDE IN COMMINUTED FRACTURE HEALING IN LOWER LIMB: A RETROSPECTIVE STUDY
}

\author{
SNEH DUDHIA ${ }^{1 *}$, KETAS MAHAJAN ${ }^{2}$ \\ ${ }^{1}$ Department of Pharmacology, Parul Institute of Medical Sciences and Research, Vadodara, Gujarat, India. ${ }^{2}$ Department of Orthopaedics, \\ Parul Sevashram Hospital, Vadodara, Gujarat, India. Email: drsneh1011@gmail.com
}

Received: 15 August 2021, Revised and Accepted: 23 September 2021

\section{ABSTRACT}

Objective: The objective of the study was to show the efficacy of teriparatide in comminuted fracture healing and improvement in overall functional recovery.

Methods: A total of 30 subjects who suffered from comminuted fractures of lower limb were selected. Subjects were categorized into two groups: Group A ( $\mathrm{n}=15)$ contained individuals with teriparatide administration and Group B $(n=15)$ contained individuals who were not administered teriparatide. $20 \mathrm{mg}$ of teriparatide was injected subcutaneously for a period of 3 months. Patients were followed up ay durations of 4, 8, and 12 weeks. Obtained data were entered into Microsoft Excel Worksheets and descriptive analysis was performed. Chi-square test was used to analyze all obtained data.

Results: Among Group A patients, the fracture healing rates were found to be significantly earlier with comparison to control group (Group B).

Conclusion: Teriparatide was observed to be effective in comminuted fracture healing and has demonstrated good clinical outcome.

Keywords: Teriparatide, Comminuted, Fracture healing.

(c) 2021 The Authors. Published by Innovare Academic Sciences Pvt Ltd. This is an open access article under the CC BY license (http://creativecommons.org/ licenses/by/4.0/) DOI: http://dx.doi.org/10.22159/ajpcr.2021v14i11.43135. Journal homepage: https://innovareacademics.in/journals/index.php/ajpcr

\section{INTRODUCTION}

Delayed fracture union or healing may be defined as a state wherein union of bone does not take place even following lapse of adequate time period from the tome of injury while non-union of fractured segments is the result of delay in union of fractures which as per the American Food and Drug Administration or FDA is a minimum period of 9 months. On the other hand, a comminuted fracture may be defined as a fracture with multiple bone fragments. It is caused by a crushing or compression force along the long axis of a bone. Although it is not possible to satisfy these criteria most of the times, a clinician makes use of clinical and radio-graphical parameters to define a non-union or delay in union of bone fractures [1]. There are various risk factors determining delayed as well as non-union of bone fractures- I. Factors specific to an individual: (a) Smoking; (b) uncontrolled diabetes; (c) infection; (d) advanced age; (e) hyperparathyroidism; (f) osteoporosis; (g) nutritional deficiencies - Vitamin D and Calcium; and (h) menopausal females II. Factors specific to fracture: (a) Minor trauma or injury; (b) sever loss of bone; (c) inadequate amount of fracture reduction and immobilization; (d) application of distraction; (e) inadequate vascular supply; (f) formation of massive hematoma; and $(\mathrm{g})$ extended use of nonsteroidal anti-inflammatory drugs [1].

Parathyroid hormone (PTH) is an important regulator of metabolism of calcium and phosphorus. There are various types of analogues of PTH that are available commercially, for example, abaloparatide and teriparatide. Both are synthetic derivatives with a polypeptide and peptide derivation with 1-84 and 1-34 constitution [2]. Serum levels of teriparatide attain peak concentration in a time interval of $30 \mathrm{~min}$ which returns to undetectable levels within few hours. Thus, there is a transient increased formation of bone at sites of fracture. Thus, by increasing thickness of bone cortex and formation of new bone, this agent causes an increase in osteogenesis, thereby promoting healing of wound [3].
Teriparatide, a synthetic polypeptide hormone, is an anabolic drug which is constituted by 1-34 amino acid fragment of PTH of humans [4]. It is a N-terminal fragment of PTH [5]. The molecular mass of Teriparatide is 4117.8 Daltons. It is synthesized using the genetically modified Escherichia coli strain. It is available commercially in solution form of injecting subcutaneously. Treatment using teriparatide has demonstrated a significant increase in volume and mineralization of callus, increase in content of mineralized bone and increase in strength and rate of union of fractures [6]. Administration of PTH or teriparatide causes enhanced expression of Osterix in mesenchymal stem cells which leads to an increase in density of bone mineralization and increase in amount of fracture callus [7].

It causes an increase of remodeling and formation of bone. It also causes a reduction in fracture risk associated with vertebrae and other bones. In addition, it has been shown to cause an accelerated healing of bone fracture due to improvement in fractured callus. This results in an increase in remodeling of bone [4]. Effects on bone also include prevention of osteoblastic apoptosis [8].

Under teriparatide therapy, formation of fracture callus occurs in abundant quantity in a circumferential manner which appears cloud-like in radiographs and computed tomography (CT) scans [4].

Teriparatide results in an improvement in micro- as well as macro-architectural characteristics of osseous tissues. It is the first ever approved drug by the US FDA agency for osteoporosis. Healing of fracture wound is a process involving multiple pathways of signals that are regulated by a variety of local as well as systemic factors. A defect or an abnormality results in impairment in healing process which leads to non-union or delay in union. Factors which cause a delay in union may be mechanical or biological in nature or may be both. This drug causes an enhancement of the Wnt/ $\beta$-catenin pathway which causes 
regulation of collagen types II and X that determines callus size [9]. The effectiveness of teriparatide therapy is significantly associated with timing of drug delivery and fracture site as fractures undergo healing process by means of various mechanisms based on fracture location. This has been attributed to mechanical stimulation of affected bone. Hence, bones which are subjected to constant loading demonstrate more responsiveness to teriparatide therapy. Teriparatide mainly demonstrates its activity by stimulating formation of bone when compared to its resorption. This is termed as "anabolic index" of the drug. Teriparatide acts by promoting proliferation and differentiation of chondro as well as osteo-progenitor mesenchymal origin stem cells, maturation of chondrocytes, bone matrix protein production and formation of osteoclasts or osteoclastogenesis $[10,11]$.

Treatment of non-union fractures using teriparatide helps patients to avoid undergoing another surgical procedure and problems associated with auto-grafts such as injury or pain to donor site or neurovascular damage [12]. Side effects include - nausea, headache, and vomiting. However, continuous administration of teriparatide increases risk of osteosarcoma, Paget's disease, and elevated levels of alkaline phosphatase. Local side effects at site of injection include - pain, itching, swelling, and erythema. It can also cause an increase in serum levels of uric acid; hence, it should be prescribed with caution in patients with renal disease impairment [12].

Absolute contraindications for usage of teriparatide are - Paget's disease of bone, both primary as well as secondary hyperparathyroidism, increased alkaline phosphatase levels, open epiphyseal ends among children, end-organ failure, pregnancy and lactation, metastatic malignancy of skeleton, and prior radiotherapy $[13,14]$. Thus, the aim of this study was to retrospectively analyze the efficacy of teriparatide administration in comminuted fracture healing in lower limbs.

\section{METHODS}

This retrospective study was performed to evaluate effects of daily administration of teriparatide on comminuted fracture wound healing in the lower limbs. The study was conducted after obtaining permission from Institutional Ethics Committee.

\section{Study type}

Retrospective study.

\section{Study place}

Shukan Multispeciality Hospital, Vadodara, Gujarat.

\section{Study duration}

2 months.

Study sample

30 patients, 15 patients in each group.

\section{Methodology}

30 patients were selected through purposive sampling and were subsequently, categorized in two groups which contained 15 patients each. Group A comprised subjects with comminuted fractures who were administered Teriparatide on a daily basis while Group B comprised patients with comminuted fractures with no teriparatide administration.

\section{Inclusion criteria}

(a) Subjects within age range of 18-65 years and (b) subjects with comminuted fractures of lower limb were included in the study.

\section{Exclusion criteria}

(a) Old and non-operated fractures; (b) patient aged $<18$ years and more than 65 years; (c) pregnant and lactating female subjects; and (d) comminuted fracture other than lower limb were excluded from the study.
Teriparatide is available as Pen injection which is easily self-administered. $20 \mathrm{mcg}$ dose was administered subcutaneously on a daily basis for a duration of 3 months.

\section{Outcome measures}

Outcome measures were dependent on - (a) Radiological features assessed by X-ray and CT scan and (b) functional outcome - weight bearing capacity. Post-operative follow-up was performed at 4,8 , and 12 weeks duration.

\section{Data analysis}

Obtained data were entered into Microsoft Excel 2007 worksheets and descriptive analysis was carried out. Chi-square test was used to analyze the obtained data and p-value $<0.05$ was considered to have statistical significance.

\section{RESULTS}

Out of 15 patients in Group A, that is, patients who were treated using teriparatide for 3 months duration, the healing rates were found to be significantly earlier $(\mathrm{p}<0.001)$ when compared to control group (Group B). Fracture union was seen to occur in nine patients with no visible fracture line in Group A. During the follow-up of Group B patients who did not receive teriparatide, six had to undergo bonegrafting surgery for achieving fracture union, six had union of fracture with visible fracture line and became pain-free following 2 months of postoperatively while three subjects demonstrated continuous pain and poor fracture healing. Subjects treated with teriparatide (mean=3.6 months) demonstrated earlier mobilization compared to those without (mean=4.5 months) ( $\mathrm{p}=0.02$ ) (Table 1 and Graph 1).

\section{DISCUSSION}

Osteoanabolic drugs are agents that aid in formation of bone hence; they are used for facilitating healing of fractures. A newer category of drugs acts by targeting sclerosis which causes inhibition of formation of bone. It is found to be expressed within osteocytes. An osteoanabolic effect may be achieved by blockage of sclerostin expression. Furthermore, bone which is formed following administration of anti-sclerosing agent is not get lost due to disuse atrophy $[15,16]$.

In our study, an enhanced fracture healing was observed in subjects treated with teriparatide on a daily basis when compared to those who did not receive the drug therapy. Our findings are corroborated by

Table 1: P-values of parameters studied on comparing both the study groups

\begin{tabular}{ll}
\hline Parameters studied & p-values \\
\hline Comparison between rates of healing & $\mathrm{p}<0.001$ \\
Mobilization period & $\mathrm{p}<0.001$ \\
\hline
\end{tabular}

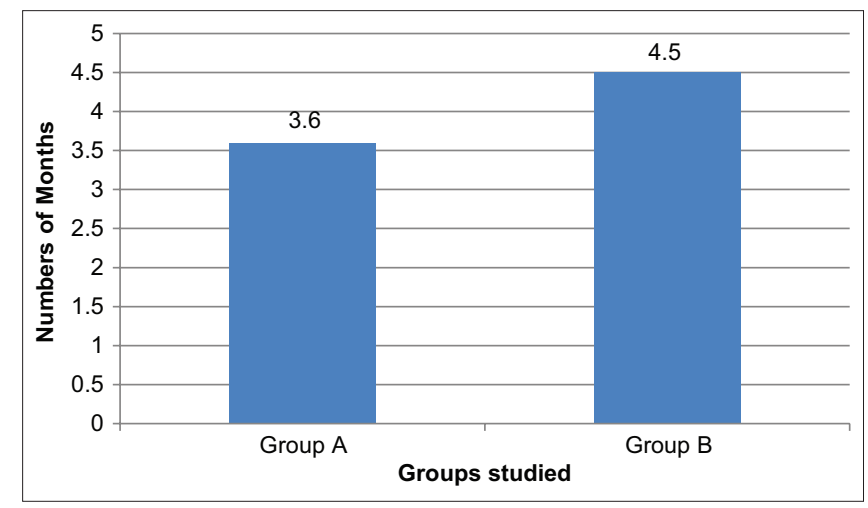

Graph 1: Demonstrating mobilization period in both groups studied 
radiological assessment as well as relatively early mobilization of the patients in treated individuals. Our findings have been supported by various investigators as described in following paragraphs.

Mishra et al. in 2019 in their prospective study on use of teriparatide on delayed union of bone fracture demonstrated enhanced healing of fracture wound [9]. Kim et al. in 2019 in their study showed that teriparatide treatment caused significant increase in Harris Hip Score $(p=0.02)$ and reduction in visual analogue pain scale score $(p=0.0005)$. The mean post-operative time required for healing of fracture was found to decrease in patient treatment with teriparatide when compared to those who did not receive this therapy (i.e., 12.1 and 14.8 weeks, respectively) [10]

Yukata et al. in 2018 in their study showed that a continuous PTH $_{1-34}$ administration or infusion led to a delay in healing of a fracture wound due to delay in maturation of callus [11]. Saraf and Munot in 2017 demonstrated that fractures which were treated with $20 \mu \mathrm{g}$ dosage of teriparatide demonstrated only signs of union associated with large amount of callus formation with significant reduction in radiographic union and early patient rehabilitation [12]. Bhandari et al. in 2016 also reported that only $15 \%$ of patients suffering from hip fractures with internal fixation who were administered teriparatide required revision surgery. Higher percentage of patients who were treated with teriparatide demonstrated early ambulation and regained prefracture movements within a duration of 12 months [16].

Kim et al. in 2015 reported effects of teriparatide administration on osteoporosis subjects. They reported formation of fracture associated callus at an early stage of healing which continued up to 8 weeks and eventually led to healing of bone fracture. Normal bone remodeling was noted on 1 year post-operative follow-up [4]. Chiang et al. in 2013 in their study demonstrated that daily subcutaneous injection of teriparatide in dosage of $20 \mathrm{mg}$ was associated with 2 to 3 times increase in expression of markers linked with remodeling of bone and healing of fracture [17].

Gomberg et al. in 2011 demonstrated that daily administration of teriparatide subcutaneously causes an increase in serum biomarker levels of formation of bone within a time span of 1 to 2 months while the markers of bone resorption demonstrate an increase in 2 to 3 months among subjects suffering from osteoporosis [18]

\section{CONCLUSION}

Teriparatide is an anabolic drug which is synthesized as a recombinant PTH analog. It has demonstrated efficacy in wound healing in osteoporotic subjects suffering from fractures, its efficacy in treating complicated fracture union has not been explored widely. This study has demonstrated that teriparatide can be used as an agent even in cases with comminuted bone fractures not only in enhancing healing period but also, in decreasing the time for mobilization or functional loading.

\section{AUTHOR CONTRIBUTION}

Both authors contributed equally to the work.

\section{CONFLICT OF INTEREST}

There were no conflicts of interest.

\section{FUNDING SOURCE}

None.

\section{REFERENCES}

1. Kundu S. Role of teriparatide in delayed union and non-union of fractures. Int J Clin Case Stud Rep 2020;2:100-10.

2. Lou S, Lv H, Li Z, Tang P, Wang Y. Parathyroid hormone analogues for fracture healing: Protocol for a systematic review and meta-analysis of randomised controlled trials. BMJ Open 2018;8:e19291.

3. Raghavan P, Christofides E. Role of teriparatide in accelerating metatarsal stress fracture healing: A case series and review of literature. Clin Med Insights Endocrinol Diabetes 2012;5:39-45.

4. Kim Y, Tanaka C, Tada H, Kanoe H, Shirai T. Radiographic features of teriparatide-induced healing of femoral fractures. Bone Rep 2015;3:11-4.

5. Yang YS, Tsou YS, Lo WC, Chiang YH, Lin JH. Teriparatide associated with fewer refractures and higher body heights of cemented vertebrae after vertebroplasty: A matched cohort study. Sci Rep 2020;10:6005.

6. Hong H, Song T, Liu Y, Li J, Jiang Q, Song Q, et al. The effectiveness and safety of parathyroid hormone in fracture healing: A meta-analysis. Clinics (Sao Paulo) 2019;74:e800.

7. Kaback LA, Soung DY, Naik A, Geneau G, Schwarz EM, Rosier RN, O'Keefe RJ, et al. Teriparatide (1-34 human PTH) regulation of osterix during fracture repair. J Cell Biochem 2008;105:219-26.

8. Babu S, Sandiford NA, Vrahas M. Use of teriparatide to improve fracture healing: What is the evidence? World J Orthop 2015;6:457-61.

9. Mishra JK, Mohapatra NC, Kar BK. Effect of daily teriperatide for delayed union of fracture neck of the femur. J OrthopTraumatol Rehabi 2019;11:49-52.

10. Kim SJ, Park HS, Lee DW, Lee JW. Short-term daily teriparatide improve postoperative functional outcome and fracture healing in unstable intertrochanteric fractures. Injury 2019;50:1364-70.

11. Yukata K, Kanchiku T, Egawa H, Nakamura M, Nishida N, Hashimoto T, et al. Continuous infusion of $\mathrm{PTH}_{1-34}$ delayed fracture healing in mice. Sci Rep 2018;8:13175.

12. Saraf HR, Munot S. Role of teriperatide in fracture healing: A prospective study. Int J Othop Sci 2017;3:445-52.

13. Hodsman AB, Baner DC, Dempster DW, Dian L, Hanley DA, Harris ST, et al. Parathyroid hormone and teriparatide for the treatment of osteoporosis: A review of the evidenc and suggested guidelines for its use. Endocr Rev 2005;26:688-703.

14. Bovbjerg P, Høgh D, Froberg L, Schmal H, Kassem M. Effect of PTH treatment on bone healing in insufficiency fractures of the pelvis: A systematic review. EFORT Open Rev 2021;6:9-14.

15. Borges JL, Freitas A, Bilezikian JP. Accelerated fracture healing with teriparatide. Arq Bras Endocrinol Metabol 2013;57:153-6.

16. Bhandari M, Jin L, See K, Burge R, Gilchrist N, Witvrouw R, et al. Does teriparatide improve femoral neck fracture healing: Results from a randomized placebo-controlled trial. Clin Orthop Relat Res 2016;474:1234-44

17. Chiang CY, Zebaze RM, Ghasem-Zadeh A, Iuliano-Burns S, Hardidge A, Seeman E. Teriparatide improves bone quality and healing of atypical femoral fractures associated with bisphosphonate therapy. Bone 2013;52:360-5

18. Gomberg SJ, Wustrack RL, Napoli N, Arnaud CD, Black DM. Teriparatide, Vitamin D, and calcium healed bilateral subtrochanteric stress fractures in a postmenopausal woman with a 13-year history of continuous alendronate therapy. J Clin Endocrinol Metab 2011;96:1627-32. 